\title{
siRNA Silencing EZH2 Reverses Cisplatin-resistance of Human Non-small Cell Lung and Gastric Cancer Cells
}

\author{
Wen Zhou ${ }^{1 \&}$, Jian Wang ${ }^{2 \&}$, Wang-Ying Man ${ }^{3}$, Qing-Wei Zhang ${ }^{1}$, Wen-Gui Xu ${ }^{2 *}$
}

\begin{abstract}
Clinical resistance to chemotherapeutic agents is one of the major hindrances in the treatment of human cancers. EHZ2 is involved in drug resistance and is overexpressed in drug-resistant cancer cell lines. In this study, we investigated the effects of EHZ2 on cisplatin -resistance in A549/DDP and AGS/DDP cells. EHZ2 mRNA and protein were found to be significantly overexpressed in A549/DDP and AGS/DDP cells, compared to parental cells. EHZ2 siRNA successfully silenced EHZ2 mRNA and protein expression. Proliferation was inhibited and drug resistance to cisplatin was improved. Flow cytometry showed that silencing of EHZ2 arrested A549/DDP and AGS/DDP cells in the G0/G1 phase, increasing apoptosis, rh-123 fluorescence intensity and caspase-3/8 activities. Silencing of EHZ2 also significantly reduced the mRNA and protein expression levels of cyclin D1 and MDR1,while up-regulating p15, p21, p27 and miR-218 in A549/DPP cells. Furthermore, silencing of EHZ2 also significantly increased the expression level of tumor suppressor factor miR-218. We also found down-regulating EHZ2 expression increased methylation in A549/DDP and AGS/DDP cells. This study demonstrates that drug resistance can be effectively reversed in human cisplatin-resistant lung and gastric cancer cells through delivery of siRNAs targeting EHZ2.
\end{abstract}

Keywords: EHZ2 - siRNA - multidrug resistance - lung cancer - gastric cancer

Asian Pac J Cancer Prev, 16 (6), 2425-2430

\section{Introduction}

Lung cancer is the most common malignant tumor and currently the cancer causing the highest mortality worldwide (Gadgeel et al., 2013). Among which, the vast majority of lung cancer is human non-small cell lung cancer (NSCLC), accounting for approximately $85 \%$ of the total number (Hensing et al., 2014). Gastric cancer is the fourth leading cancer in the world (Shi et al., 2014) and its prevalence ranks the second in China (Zhang et al., 2010).

Chemotherapy is the main treatment method for these 2 types of cancers either as neoadjuvant therapy (i.e. pre-operative treatment), as adjuvant therapy (i.e. postoperative treatment), or as palliative therapy for patients with inoperable advanced cancers. Platinum drugs are an important class of chemotherapeutic drugs for cancers, among which, cisplatin is one of the most representative drugs (Hato et al., 2014). However, lung cancer tends to develop resistance to cisplatin during chemotherapy, leading to treatment failure. In contrast, gastric cancer is often insensitive to cisplatin at the beginning of treatment. In the past 2 decades, targeted therapy has made great success with cancer genomics and molecular biology; taking the small-molecule drugs for the treatment of NSCLC for examples: epidermal growth factor receptor (EGFR) inhibitors gefitinib and erlotinib (Jang et al., 2014), EGFR monoclonal antibody cetuximab (Janjigian et al., 2014), vascular endothelial growth factor (VEGF) monoclonal antibody bevacizumab (Powell et al., 2013); the examples for the treatment of gastric cancer include human epidermal growth factor receptor 2 (Her 2) monoclonal antibody trastuzumab (Zheng et al., 2014) and vascular endothelial cell growth factor receptor (VEGFR) monoclonal antibody ramucirumab (Wadhwa et al., 2014). But, these targeted drugs have very clear therapeutic indications that can only benefit patients with positive specific biomarkers. Although these drugs can improve the response rate, prolong the progression-free survival and improve the quality of life in these patients, they have limited impact on the overall survival. Therefore, the relatively economic conventional chemotherapeutic drugs still play very important roles in first-line treatment of these cancers.

In order to resolve drug resistance of chemotherapeutic drugs, develop new targeted drugs, and improve the

${ }^{I}$ Tianjin Key Laboratory on Technologies Enabling Development of Clinical Therapeutics and Diagnostics (Theranostics), School of Pharmacy, Tianjin Medical University, ${ }^{2}$ Department of Molecular Imaging and Nuclear Medicine, Tianjin Medical University Cancer Institute and Hospital, National Clinical Research Center for Cancer, Key Laboratory of Cancer Prevention and Therapy, Tianjin, ${ }^{3}$ Department of Hematology and Oncology, Hebei United University Affiliated Hospital, Tangshan, China ${ }^{\circledR}$ Equal contributors *For correspondence:wengui_xutj@163.com 


\section{Wen Zhou et al}

clinical benefits of cancer patients, it is necessary to continue the search for new biomarkers. EZH2, a catalytic subunit of polycomb repressive complex (PRC2), can either inhibit gene transcription by methylating lysine 27 on histone 3 (Fujii et al., 2008; Popovic et al., 2014) or directly inhibit gene activity by methylating DNA bases (Liu et al., 2014). It has been found in NSCLC (Behrens et al., 2013) and gastric cancer (Choi et al., 2013; He et al., 2013) that EZH2 over-expression is positively correlated with poor prognosis of the patients. In addition, some studies showed that EZH2 played positive roles in cisplatin-resistant ovarian cancers (Hu et al., 2010; Tamgue et al., 2013) and 5-Fu-resistant liver cancers (Zhang et al., 2013) This study not only investigated the effects of EZH2 on cisplatin resistance in NSCLC and gastric cancer but also preliminarily probed its molecular mechanisms.

\section{Materials and Methods}

\section{Main reagents and instruments}

Human lung cancer A549 cell line and gastric cancer AGS cell line were purchased from ATCC; MTS, Rh-123 and RT-PCR kits were purchased from Promega; cisplatin was purchased from Sigma-Aldrich; EZH2 siRNA and related reagents were purchased from Cell Signaling Technology; PI cell cycle determination kit, Annexin V-FITC/PI kit and Caspase-3/8 activity kit were purchased from BD; various monoclonal antibodies were purchased from SantaCruz; ECL immunoblotting substrate kit was purchased from Millipore; flow cytometry: BD; microplate reader: Thermo; PCR instrument: Thermo.

\section{Cell culture and induction of drug-resistance cell line}

Both A549 and AGS cells were maintained in F12K (containing 10\% fetal bovine serum) and cultured in an incubator under $37^{\circ} \mathrm{C}, 5 \% \mathrm{CO}_{2}$ and saturated humidity condition. The cells were digested with $0.25 \%$ trypsinEDTA for passaging. Cells in logarithmic growth phase were used in all experiments. In order to induce drugresistance cell line, the primary cells were cultured with sub-lethal dose (the initial concentration was $1 / 10$ of the $\left.\mathrm{IC}_{50}\right)$ of cisplatin, which was gradually escalated until $\mathrm{IC}_{50}$ of cisplatin for cells was more than 10 times the initial value.

\section{siRNA silencing EZH2 expression}

The cells were cultured in $10 \mathrm{~cm}$ culture dishes until reaching approximately $70 \%$ confluence. The siRNA reagent was prepared in accordance with procedures described in the kit instructions; the working concentration of siRNA was $100 \mathrm{nM}$. In $48 \mathrm{~h}$ after transfection, the culture was changed to regular medium to continue the culture for $24 \mathrm{~h}$ prior to subsequent experiments. The number of cell subcultures used for general experiments did not exceed 2 passages after siRNA treatment in order to avoid attenuation of the silencing effect. The silencing of EZH2 was assessed using Western blotting and RT-PCR.

Determination of cell sensitivity to cisplatin using MTS assay
The cells in logarithmic growth phase, $5 \times 10^{4}$ cells/ $\mathrm{ml}$, were seeded in 96-microwell plates, $100 \mu \mathrm{l} /$ well, and cultured overnight until cell adhesion. Different concentrations of cisplatin were added to continue the culture for $72 \mathrm{~h}$. After removing the medium, MTS was added in accordance with the kit instructions to continue the culture for $4 \mathrm{~h}$. Finally, the OD was measured at 490nm wavelength using a microplate reader. The inhibition rate of the drug on these cells was calculated as follows: Inhibit rate $=(1$-experimental group OD/control group OD) $\times 100 \%$. The inhibition curve was plotted and fitted using cisplatin concentration as the abscissa and inhibition rate was the ordinate to obtain the $\mathrm{IC}_{50}$ value. The reversion fold $(\mathrm{RF})=\mathrm{IC}_{50}$ (silencing group)/IC $\mathrm{I}_{50}$ (control group).

Determination of intracellular Rh-123 content, cell cycle, apoptosis and Caspase-3/8 activity using flow cytometry

The cells in logarithmic growth phase were incubated with $10 \mu \mathrm{M}$ Rh-123 staining solution for $1 \mathrm{~h}$. Then, the cells were collected to determine the intracellular fluorescence intensity of Rh-123 using flow cytometry in order to reflect the intracellular Rh-123 content. The cell cycle was determined with PI staining method. After the cells had been treated with $5 \mu \mathrm{M}$ cisplatin for $24 \mathrm{~h}$, the cells were collected to determine the cell cycle using method described in the kit instructions. Apoptosis was determined using Annexin V-FITC/PI doubling-staining technique. After the cells had been treated with $20 \mu \mathrm{M}$ cisplatin for $24 \mathrm{~h}$, the cells were collected to determine apoptosis using method described in the kit instructions. After the cells had been treated with cisplatin in the same way, intracellular Caspase- 3 and Caspase- 8 activities were determined using PE-labeled anti-activated Caspase-3 and Caspase- 8 antibodies and detected using flow cytometry in accordance with the kit instructions.

Determination of intracellular EZH2, cyclin D1, p15, p21, added MRP1, GST- $\pi, P$-gp, Survivin, Livin and p27 expression levels using Western blotting

The cells in logarithmic growth phase were lysed to extract the proteins from cell lysate. The proteins were separated in $12 \%$ SDS-PAGE and transferred onto a PVDF membrane. Different antibodies were used to determine the target proteins $\left(4^{\circ} \mathrm{C}\right.$, overnight $)$. After washing off the primary antibodies, the membrane was incubated with HRP-conjugated secondary antibody for $1 \mathrm{~h}$. After washing, ECL kit was used to develop the immunoreactive bands using $\beta$-actin as the internal reference.

Determination of EZH2, cyclin D1, p15, p21, added MRP1, GST-л, P-gp, Survivin, Livin and p27 mRNA levels, and miRNA-218 level in A549/DPP tumor cells using RT-PCR technique

The total RNA was extracted using Trizol method. Reverse transcription was carried out using Real-Time PCR kit to obtain the cDNA. The cDNA $(1 \mu \mathrm{l})$ was amplified by PCR ( pre-denaturation step at $95^{\circ} \mathrm{C}$ f or 5 min followed by 40 cycles of $95^{\circ} \mathrm{C}$ f or $30 \mathrm{~s}, 60^{\circ} \mathrm{C}$ f or $30 \mathrm{~s}$, and $72^{\circ} \mathrm{C}$ f or $30 \mathrm{~s}$; then $72^{\circ} \mathrm{C}$ f or $10 \mathrm{~min}$ ). Sequences of primers used are as follows: $\beta$-actin upstream, 5'-TTAGCTGTGCTCGCGCTACTCTCTC-3', 
$\begin{array}{lllllllllll}\mathrm{d} & \mathrm{o} & \mathrm{w} & \mathrm{n} & \mathrm{s} & \mathrm{t} & \mathrm{r} & \mathrm{e} & \mathrm{a} & \mathrm{m}\end{array}$ 5'-GTCGGATTGATGAAACCCAGACACA-3'; EZH2 upstream, 5' - CTTCTGCTGTGCCCT TATC -3', downstream, 5' - CCGTCCACCTCGGAAAT -3'; cyclin D1 upstream, 5' - GGCGGA GGAGAACAAACAGA -3', downstream, 5' TGGCACAAGAGGCAACGA -3'; p15 upstream, 5'- GATGTGCAAGCGACGACAGA -3', downstream, 5'- GAGCAAAGGCCAGCA TCCT -3';p21 upstream, 5'- CCTCATCCCGTGTTCTCCTTT -3', downstream, 5'GTACCACCC AG CGGACAAGT -3';MDR1 upstream, 5' - AAATTGGCTTGACAAGTTGTATATGG -3', downstream, 5'- CACCAGCATCATGAGAGGAAGTC -3';MRP1 upstream, 5' - TCTACCTCCT GTGGCTGAATCTG - 3', downstream, 5' CCGATTGTCTTTGCTCTTCATG -3'; GST- $\pi$ upstream, 5'- TGGGCATCTGAAGCCTTTTG -3', downstream, 5'- GATCTGGTCACCCACG ATGAA -3'; Survivin upstream, 5' - CGAGGCTGGCTTCATCCACT -3', downstream, 5' - ACG GCGCACTTTCTTCGCA -3'; Livin upstream, 5'- GTCCCTGCCTCTGGGTAC -3', downstream, 5'- CAGGGAGCCCACTCTGCA -3'; p27 upstream, 5'- CTGCAACCGACGATTC TTCTACT -3', downstream, 5'- GGGCGTCTGCTCCACAGA -3'; The PCR primer of miRNA-218 was purchased from Applied Biosystems. the fold changes were calculated by the delta-delta $\mathrm{Ct}$ method. All experiments were performed in three biological replicates.

\section{Determination of MDR1 gene methylation level}

Phenol-chloroform method was used to extract total DNA from the cells. CpGenome DNA Modification kit (purchased from Chemicon) was used for sodium bisulfate modification of the DNA. Then, the methylation level of MDR1 gene was determined using nested PCR technique; the primers used in the first round of PCR were upstream: 5'-GGAAGTTAGAATATTTTTTTTGGAAAT-3' $\begin{array}{lllllllllllll} & \mathrm{n} & \mathrm{d} & \mathrm{d} & \mathrm{o} & \mathrm{w} & \mathrm{n} & \mathrm{s} & \mathrm{t} & \mathrm{r} & \mathrm{e} & \mathrm{a} & \mathrm{m}\end{array}$ 5'-ACCTCTACTTCTTTAAACTTAAAAAAACC-3'. The primers used in the second round of PCR to determine the methylation sequence were upstream: 5'-CGAGGAATTAGTATTTAGTTAATTCGGGTCGG-

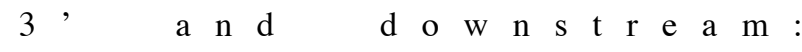

A

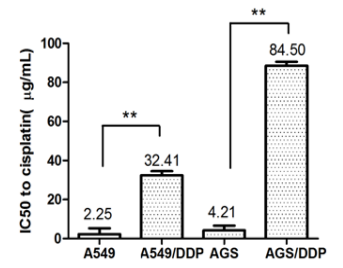

B

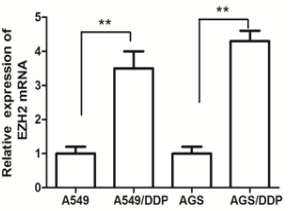

Figure 1. EZH2 Expression Level was Significantly Up-regulated in A549/DDP and AGS/DDP Cells. EZH2 mRNA(A) and protein(B) expression levels in A549/DDP and AGS/DDP cells were detected by Real-time PCR and western blotting, respectively. The statistical significance was considered as $* p<0.05$ and $* * p<0.01$ where compared with control
5'-ACTCAACCCACGCCCCGACG-3'. The primers used for the determination of non-methylation sequence were upstream: 5'-TGAGGAATTAGTATTTAGTTA A T T TGGG T TGG-3, and downstream: 5'-ACTCAACCCACACCCCAACA-3'. The specific test methods referred to the literature (Zhang et al., 2013).

\section{Statistical analysis}

The experimental data were expressed as mean \pm standard deviation. SPSS13.0 software was used for the analysis. One-way ANOVA was used for comparisons; $p<0.05$ indicated that the difference was statistically significant.

\section{Results}

EZH2 expression level in cisplatin drug-resistant cell line is significantly up-regulated

After gradual induction, the drug-resistant cell lines A549/DDP and AGS/DDP were successfully obtained. As showed Figure 1, with MTS assay, their respective drug resistance (by comparing the $\mathrm{IC}_{50}$ ) was 16 and 21 times of the original cell lines. Both WB and RT-PCR showed that the EZH2 level was significantly higher in these drugresistant cells than in the original cell lines.

siRNA significantly down-regulates EZH2 expression level in drug-resistant cells and reverses cisplatin drug resistance

In $72 \mathrm{~h}$ after siRNA transfection, both WB and RTPCR results showed that EZH2 expression level in A549/ DDP and AGS/DDP cell significantly decreased, while control siRNA has no effect on EZH2 expression. MTS assay result showed that, after EZH2 silencing, cisplatin resistance of A549/DDP and AGS/DDP was significantly reversed to varying degrees (Figure 2).

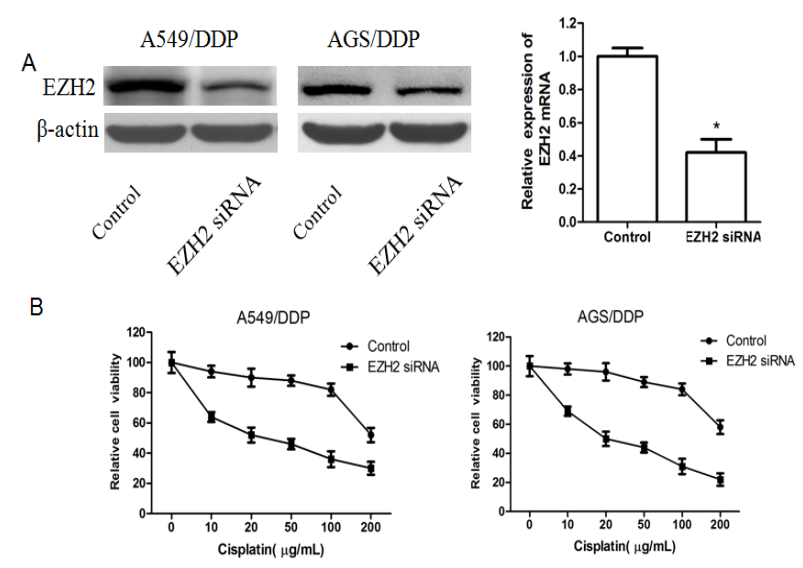

Figure 2. Silencing of EZH2 Significantly Reverses Cisplatin Drug Resistance in A549/DDP and AGS/DDP Cells. A: After transfected with EZH2 siRNA,EZH2 Mrna and protein expression levels in A549/DDP and AGS/DDP cells were detected by Real-time PCR and western blotting, respectively. B: After transfected with EZH2 siRNA,the cell viabilities of A549/ DDP and AGS/DDP cells were detected by MTS assay. The statistical significance was considered as $* p<0.05$ and $* * p<0.01$ where compared with control 
EZH2 silencing increases Rh-123 content in drug-resistant cells, arrests the cell cycle in G0/G1 phase, and increases cisplatin-induced apoptosis rate and Caspase-3/8 activity

The flow cytometric results were shown in Figure 3, after EZH2 silencing, the intracellular Rh-123 fluorescence intensity increased by 3.28 fold and 4.06 fold when compared with that in the control group. EZH2 silencing alone was sufficient to arrest A549/DDP and AGS/DDP cell cycles in G0/G1 phase. Cell cycle arrest effect was more prominent after treatment with $5 \mu \mathrm{M}$ cisplatin for 24h. In addition, EZH2 silencing itself did not have clear impact on the apoptosis of drug-resistant cells; but, after treatment with $20 \mu \mathrm{M}$ cisplatin for $24 \mathrm{~h}$, the apoptosis rate was $8.8 \%$ and $17.4 \%$ in A549/DDP control group and the silencing group, respectively, the apoptosis rate was $11.1 \%$ and $16.4 \%$ for AGS/DDP groups; the differences had statistical significance. Similarly, after treatment with cisplatin, the contents of activated Caspase- 3 and Caspase- 8 were significantly higher in silencing group.

EZH2 silencing down-regulates cyclin D1 and MDR1 expression while up-regulates p15,p21,p27 and miR-218 expression in A549/DPP cells

WB results showed that, when compared with the

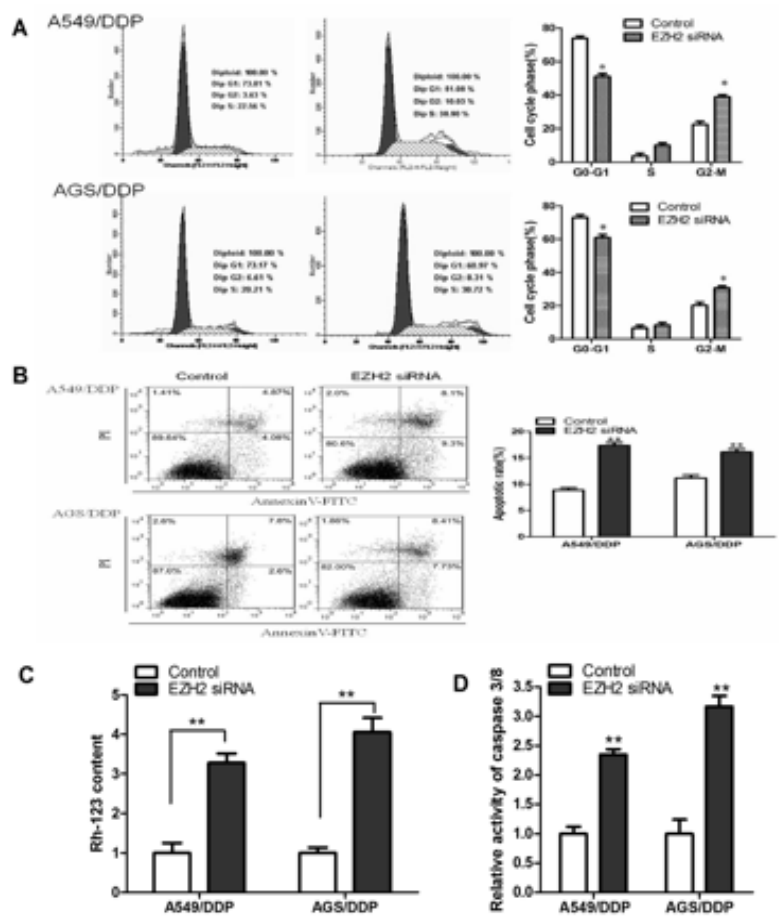

Figure 3. EZH2 Silencing Induced Cell-cycle Arreste and Opoptosis in A549/DDP and AGS/DDP Cells. A: Cells were transfected with EZH2 siRNA for 24h.PI staining was used to analyze the cell cycle distribution; B: Cells were transfected with EZH2 siRNA for 24h, and then cells as assessed by Annexin V-fluorescein isothiocyanate and propidium iodide (PI) double staining and fluorescence-activated cell sorter analysis; C: The intracellular rhodamine 123 accumulation in A549/DDP and AGS/DDP cells were detected by flow cytometry after transfected with EZH2 siRNA for 24.The statistical significance was considered as $* p<0.05$ and $* * p<0.01$ where compared with control; D: Intracellular Caspase-3 and Caspase- 8 activities in A549/DDP and AGS/DDP cells were detected by flow cytometry after transfected with EZH2 siRNA for 24. Statistical significance was considered as $* p<0.05$ and $* * p<0.01$ control group, the expression level of cyclin D1, a check-point control protein of G0/G1 phase, significantly decreased in drug-resistant cells after EZH2 silencing. The expression of multi-drug-resistant protein MDR1 also decreased significantly. In contrast, the expression of cell cycle negative regulators such as p15, p21 and p27 increased significantly. The study also found that the expression level of tumor suppressor factor miR-218 significantly increased after EZH2 silencing (Figure 4).

\section{MDR1 gene methylation level after EZH2 silencing}

To further investigate the mechanisms of EZH2mediated regulation of gene expression, we determined the methylation level of MDR1. As shown by the electrophoretic analysis of nested PCR products, in drugresistant cell lines A549/DDP and AGS/DDP, MDR1 gene methylation band was darker; after EZH2 silencing, this band became significantly lighter, suggesting that EZH2 is able to regulate the related genes through this epigenetic approach, the methylation (Figure 5).

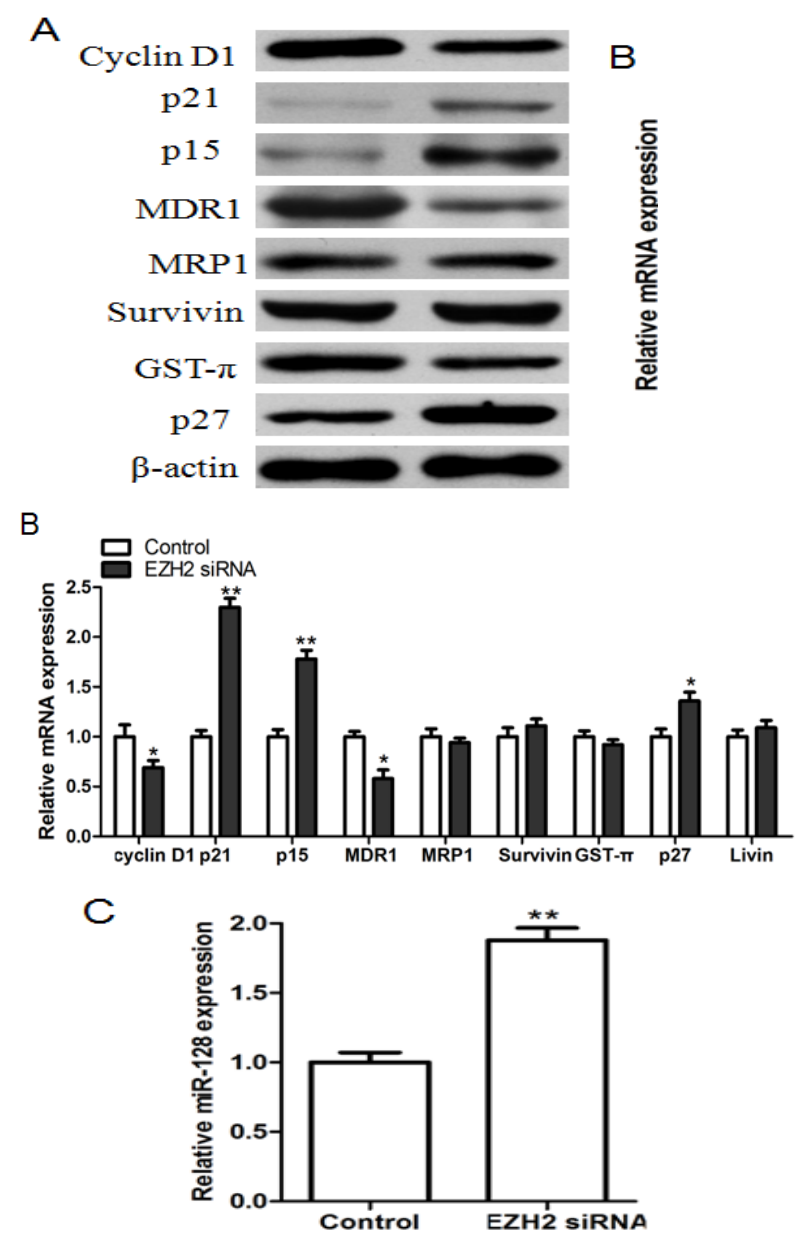

Figure 4. Silencing of EZH2 Affects mRNAs and Proteins of Associated with Proliferation and Apoptosis in A549/DDP and AGS/DDP Cells. A: A549/DDP cells were transfected with EZH2 siRNA for $72 \mathrm{~h}$, and then cells were collected. The total cell lysates of suspended were analyzed by western blotting to examine the expression of the cell proliferation and apoptosis proteins(EZH2, cyclin D1, p15, p21, added MRP1, GST- $\pi$, P-gp, Survivin, Livin and p27).The mRNA expression levels of above gene(B) and miR-218 (C) were dected by real-time PCR. The statistical significance was considered as ${ }^{*} p<0.05$ and ${ }^{*} p<<0.01$ where compared with control 


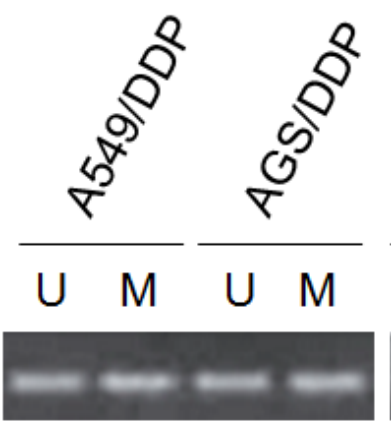

Control
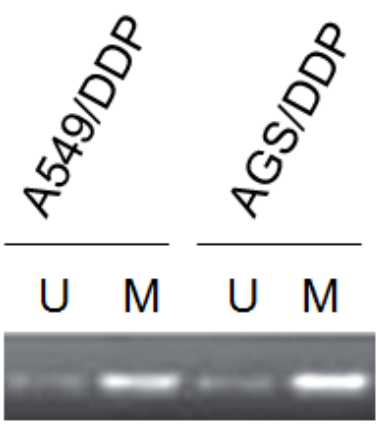

EZH2 siRNA
Figure 5. Methylation-specific PCR Analysis of the Methylation Status of MDR1. PCR products specific for unmethylation (U) and methylation (M) were analysed in 3\% agarose gels. The $\mathrm{U}$ and $\mathrm{M}$ bands were almost equally visible in the control groups, while the $\mathrm{M}$ bands were markedly brighter than the U bands in the siEZH2 groups in A549/DDP and AGS/ DDP cells

\section{Discussion}

Although some studies have shown that EZH2 plays important roles not only in cancer development and progression but also in chemotherapeutic drug resistance of some types of tumors, the studies on its potential roles in cisplatin resistance of NSCLC and gastric cancer are few. In this study, we first constructed two cisplatin-resistant cancer cell lines, namely A549/DDP and AGS/DDP. We found that EZH2 expression level was significantly higher in drug-resistant cells than in the original cell lines. After further silencing EZH2 using siRNA, cisplatin sensitivity of the drug-resistant cells increased significantly. These results strongly suggest that EZH2 plays an important role in cisplatin resistance of NSCLC and gastric cancer. EZH2, as a methyltransferase, can regulate gene expression indirectly by methylating the histone or directly by methylating the DNA. This gene regulation manner belongs to the category of epigenetics and cannot be detected with conventional genomic study method, namely the DNA sequencing. However, studies over the past decade have discovered its key roles in tumors (Yamaguchi et al., 2014). Currently, some selective small-molecule EZH2 inhibitors with significant anti-tumor effects have been discovered and entered clinical trials (Avan et al., 2012; Garapaty et al., 2013). Notably, studies on the roles of EZH2 in tumor resistance to chemotherapeutic drugs and the mechanisms of actions have very important significance because they can provide the basis for clinical application of EZH2 inhibitor and chemotherapeutic drug combinations.

The two primary mechanisms responsible for tumor cell drug resistance usually include over-expression of multi-drug resistance genes to increase drug efflux resulting in reduction of intracellular drug concentration, and resistance to drug-induced apoptosis (Zhang et al., 2013). We hypothesized that EZH2 may also reverse A549/DDP and AGS/DDP drug resistance by regulating these two drug-resistant mechanisms. Accordingly, we carried out relevant studies to test this hypothesis. The results showed that EZH2 silencing increased intracellular
Rh-123 concentration and enhanced apoptosis. Western blotting results showed that MDR1 expression was significantly down-regulated after EZH2 silencing. This protein belongs to the ABC superfamily capable of pumping chemotherapeutic drugs out of the cells (Ansbro et al., 2013). Down-regulating the expression of these proteins can increase the intracellular concentration of chemotherapeutic drugs, which is consistent with our observation on elevated intracellular Rh-123 level. This result is also consistent with the results observed by Zhang et al. (2013) and Zhu et al. (2014) in 5-Furesistant hepatoma cells. Both Annexin V/PI staining and Caspase- $3 / 8$ activity assay results suggest that silencing EZH2 is able to increase cisplatin-induced tumor apoptosis rate, but EZH2 silencing itself did not induce significant apoptosis, suggesting that EZH2 may not be directly involved in the regulation of apoptosis; the higher cisplatin-induced apoptosis rate in EZH2 silencing group may be related to the increased intracellular cisplatin concentration resulting from $\mathrm{EZH} 2$ silencing.

In addition, we examined the effects of EZH2 on cell cycle. In mammalian cells, CDK4/6-cyclin D and CDK2-cyclin E complexes are the primary cell cycle G1/S checkpoint factors controlling whether cells leave G1 phase to enter S phase and start DNA synthesis(Robson et al., 1999) While, p15, p21 and p27 are negative regulators of these two complexes and therefore the cell cycle inhibitors (Robson et al., 1999; Sherr et al., 1999). Our WB results showed that after EZH2 silencing, cyclin D1 expression level was down-regulated while p15, p21 and p27 expression level were up-regulated, suggesting that $\mathrm{EZH} 2$ regulates cell cycle through coordinated regulation of a variety of cell cycle-related factors.

RT-PCR results showed that EZH2 regulated all the above proteins at mRNA level, which is consistent with the nature of epigenetic regulation of EZH2. To further confirm the gene regulation mode of EZH2 in A549/ DDP and AGS/DDP cells, we determined MDR1 gene methylation level and found that the degree of MDR1 gene methylation increased after EZH2 silencing. Because gene methylation is generally regarded as a gene silencing marker (Suzuki et al., 2014), this result is consistent with the finding that EZH2 silencing down-regulated MDR1 expression. We also determined miRNA-218 expression level; the recent finding has showed that EZH2 can silence this microRNA to promote the development of pancreatic ductal adenocarcinoma (Li et al., 2011). The miR-218, a tumor suppressor gene, plays a role in gastric cancer, oral squamous cell carcinoma, and medulloblastoma (Tie et al., 2010; Peng et al., 2014;Uesugi et al., 2011; Venkataraman et al., 2013). Our study showed that EZH2 silencing was also able to increase miR-218 level in A549/DDP and AGS/DDP cells to further exert tumor suppressor actions, suggesting that EZH2 can regulate cisplatin resistance indirectly.

In summary, this study shows that silencing EZH2 can reverse cisplatin resistance of NSCLC and gastric cancer cells; its mechanisms of action may be related to the down-regulation of multi-drug resistance gene expression, inhibiting drug efflux, promoting apoptosis, inhibiting cell cycle, and activating certain tumor 


\section{Wen Zhou et al}

suppressor factor activities. This study will provide the basis for the development of EZH2 targeted drugs and the combination with chemotherapeutic drugs to improve the patients' benefits.

\section{References}

Ansbro MR, Shukla S, Ambudkar SV, et al (2013). Screening compounds with a novel high-throughput ABCB1-mediated efflux assay identifies drugs with known therapeutic targets at risk for multidrug resistance interference. PLoS One, $\mathbf{8}$, 60334.

Avan A, Crea F, Paolicchi E, et al (2012). Molecular mechanisms involved in the synergistic interaction of the EZH2 inhibitor 3-deazaneplanocin A with gemcitabine in pancreatic cancer cells. Mol Cancer Ther, 11, 1735-46.

Behrens C, Solis LM, Lin H, et al (2013). EZH2 protein expression associates with the early pathogenesis, tumor progression, and prognosis of non-small cell lung carcinoma. Clin Cancer Res, 19, 6556-65.

Choi JH, Song YS, Yoon JS, et al (2010). Enhancer of zeste homolog 2 expression is associated with tumor cell proliferation and metastasis in gastric cancer. APMIS, 118, 196-202.

Fujii S, Ochiai A (2008). Enhancer of zeste homolog 2 downregulates E-cadherin by mediating histone $\mathrm{H} 3$ methylation in gastric cancer cells. Cancer Sci, 99, 738-46.

Gadgeel SM (2013). New targets in non-small cell lung cancer. Curr Oncol Rep, 15, 411-23.

Garapaty-Rao S, Nasveschuk C, Gagnon A, et al (2013). Identification of EZH2 and EZH1 small molecule inhibitors with selective impact on diffuse large B cell lymphoma cell growth. Chem Biol, 20, 1329-39.

Hato SV, Khong A, de Vries IJ, et al (2014). Molecular pathways: the immunogenic effects of platinum-based chemotherapeutics. Clin Cancer Res, 20, 2831-7.

He LJ, Cai MY, Xu GL, et al (2013). Prognostic significance of overexpression of $\mathrm{EZH} 2$ and $\mathrm{H} 3 \mathrm{k} 27 \mathrm{me} 3$ proteins in gastric cancer. Asian Pac J Cancer Prev, 13, 3173-8.

Hensing T, Chawla A, Batra R, et al (2014). A personalized treatment for lung cancer: molecular pathways, targeted therapies, and genomic characterization. Adv Exp Med Biol, 799, 85-117.

$\mathrm{Hu}$ S, Yu L, Li Z, et al (2010). Overexpression of EZH2 contributes to acquired cisplatin resistance in ovarian cancer cells in vitro and in vivo. Cancer Biol Ther, 10, 788-95.

Jang WJ, Park B, Jeong GS, et al (2014). SB365, Pulsatilla saponin D, suppresses the growth of gefitinib-resistant NSCLC cells with Met amplification. Oncol Rep, 32, 2612-8.

Janjigian YY, Smit EF, Groen HJ, et al (2014). Dual inhibition of EGFR with afatinib and cetuximab in kinase inhibitorresistant EGFR-mutant lung cancer with and without T790M mutations. Cancer Discov, 4, 1036-45.

Li CH, To KF, Tong JH, et al(2013). Enhancer of zeste homolog 2 silences microRNA-218 in human pancreatic ductal adenocarcinoma cells by inducing formation of heterochromatin. Gastroenterology, 144, 1086-97.

Liu T, Hou L, Huang Y(2014).EZH2-specific microRNA-98 inhibits human ovarian cancer stem cell proliferation via regulating the pRb-E2F pathway. Tumour Biol, 35, 7239-47.

Peng SC, Liao CT, Peng CH, et al (2014). MicroRNAs MiR-218, MiR-125b, and Let-7g predict prognosis in patients with oral cavity squamous cell carcinoma. PLoS One, 9, 102403.

Popovic R, Martinez-Garcia E, Giannopoulou EG, et al (2014). Histone methyltransferase MMSET/NSD2 alters EZH2 binding and reprograms the myeloma epigenome through global and focal changes in H3K36 and H3K27 methylation.
PLoS Genet, 10, 1004566.

Powell SF, Beitinjaneh A, Tessema M, et al (2013). Phase II study of topotecan and bevacizumab in advanced, refractory non--small-cell lung cancer. Clin Lung Cancer, 14, 495-501.

Robson CN, Gnanapragasam V, Byrne RL, et al (1999). Transforming growth factor-beta1 up-regulates p15, p21 and p27 and blocks cell cycling in G1 in human prostate epithelium. J Endocrinol, 160, 257-66.

Sherr CJ, Roberts JM(1999).CDK inhibitors: positive and negative regulators of G1-phase progression. Genes Dev, 13, 1501-12.

Shi J, Qu YP, Hou P (2014). Pathogenetic mechanisms in gastric cancer. World J Gastroenterol, 20, 13804-13819.

Suzuki M, Ikeda K, Shiraishi K, et al (2014).Aberrant methylation and silencing of IRF8 expression in non-small cell lung cancer. Oncol Lett, 8, 1025-30.

Sneeringer CJ, Scott MP, Kuntz KW, et al (2010).Coordinated activities of wild-type plus mutant EZH2 drive tumorassociated hypertrimethylation of lysine 27 on histone $\mathrm{H} 3$ (H3K27) in human B-cell lymphomas. Proc Natl Acad Sci U S A, 107, 20980-5.

Tamgue O, Chai CS, Hao L, et al (2013). Triptolide inhibits histone methyltransferase EZH2 and modulates the expression of its target genes in prostate cancer cells. Asian Pac J Cancer Prev, 10, 5663-9.

Tie J, Pan Y, Zhao L, et al (2010). MiR-218 inhibits invasion and metastasis of gastric cancer by targeting the Robo1 receptor. PLoS Genet, 6, 1000879.

Uesugi A, Kozaki K, Tsuruta T, et al (2011).The tumor suppressive microRNA miR-218 targets the mTOR component Rictor and inhibits AKT phosphorylation in oral cancer. Cancer Res, 71, 5765-78.

Venkataraman S, Birks DK, Balakrishnan I, et al (2013). MicroRNA 218 acts as a tumor suppressor by targeting multiple cancer phenotype-associated genes in medulloblastoma. J Biol Chem, 288, 1918-28.

Wadhwa R, Elimova E, Shiozaki H, et al (2014). Anti-angiogenic agent ramucirumab: meaningful or marginal? Expert Rev Anticancer Ther, 14, 367-79.

Yamaguchi H, Hung MC (2014). Regulation and Role of EZH2 in Cancer. Cancer Res Treat, 46, 209-22.

Zhang D, Fan D (2010). New insights into the mechanisms of gastric cancer multidrug resistance and future perspectives. Future Oncol, 6, 527-37.

Zhang JY, Lin MT, Yi T, et al (2013). Apoptosis sensitization by Euphorbia factor L1 in ABCB1-mediated multidrug resistant K562/ADR cells. Molecules, 18, 12793-808.

Zhang Y, Liu G, Lin C, et al (2013). Silencing the EZH2 gene by RNA interference reverses the drug resistance of human hepatic multidrug-resistant cancer cells to 5-Fu. Life Sci, 92, 896-902.

Zheng L, Tan W, Zhang J, et al (2014). Combining trastuzumab and cetuximab combats trastuzumab-resistant gastric cancer by effective inhibition of EGFR/ErbB2 heterodimerization and signaling. Cancer Immunol Immunother, 63, 581-6.

Zhu Y, Liu XJ, Yang P, et al (2014). Alkylglyceronephosphate synthase (AGPS) alters lipid signaling pathways and supports chemotherapy resistance of glioma and hepatic carcinoma cell lines. Asian Pac J Cancer Prev, 7, 3219-26. 